\title{
Artificial Protein-Responsive Riboswitches Upregulate Non-AUG Translation Initiation in Yeast
}

Fumihiro Horie, Kei Endo*, and Koichi Ito*

Department of Computational Biology and Medical Sciences, Graduate School of Frontier Sciences, The University of Tokyo, 5-1-5 Kashiwanoha, Kashiwa-shi, Chiba 277-8562, Japan

Correspondence to:

Email: kei-endo@k.u-tokyo.ac.jp (K.E.), itokoichi@k.u-tokyo.ac.jp (K.I.)

\section{Supporting Information}

\section{Contents}

Text S1. The sequences of the non-AUG ON switches shown in Figure 2 ............................................ 2

Text S2. The sequences of the ligand proteins used in this study. ......................................................... 3

Text S3. The sequence of C-terminal protein tag fused in Figure 3E and 3F. ...................................... 6

Text S4. The 5' leader sequence of the dCas9-driven non-AUG ON switch w/o NCC.............................6

Figure S1. Western blotting analysis of trans-acting ligand proteins. ..................................................... 7

Figure S2. The working model for the MCP-driven non-AUG ON switch. ..........................................

Figure S3. Predicted secondary structure and thermostability of the MS2 stem-loop and the sgRNA for

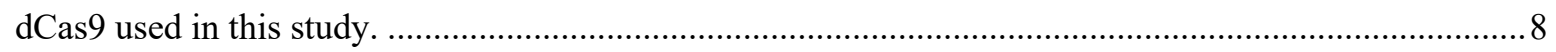

Table S1. List of plasmid sets used in this study................................................................................

Table S2. The primers used for constructing the non-AUG ON switches in Figure 4........................... 12

Table S3. The primers used for constructing the non-AUG ON switches in Figures 5 and 6 ................ 13 


\section{Text S1. The sequences of the non-AUG ON switches shown in Figure 2.}

The constructs of the non-AUG ON switches, composed of the 5' leader sequence followed by HIS3 or Luc2 reporter gene lacking the authentic AUG codon, are inserted into the BamHI-SalI site of p416TEF. Their sequences are as follows:

A) BamHI site - original 5' leader - MS2 stem-loop - HIS3( $\triangle A U G)$ - Sall site 5' -GGATCCCTGCAGACATATACTGATACGATTGATCAGGTCTCGGTGAGGATCACCCATCGAAGATCC ACAGAGCAGAAAGCCCTAGTAAAGCGTATTACAAATGAAACCAAGATTCAGATTGCGATCTCTTTAAAGG GTGGTCCCCTAGCGATAGAGCACTCGATCTTCCCAGAAAAAGAGGCAGAAGCAGTAGCAGAACAGGCCAC ACAATCGCAAGTGATTAACGTCCACACAGGTATAGGGTTTCTGGACCATATGATACATGCTCTGGCCAAG CATTCCGGCTGGTCGCTAATCGTTGAGTGCATTGGTGACTTACACATAGACGACCATCACACCACTGAAG ACTGCGGGATTGCTCTCGGTCAAGCTTTTAAAGAGGCCCTAGGGGCCGTGCGTGGAGTAAAAAGGTTTGG ATCAGGATTTGCGCCTTTGGATGAGGCACTTTCCAGAGCGGTGGTAGATCTTTCGAACAGGCCGTACGCA GTTGTCGAACTTGGTTTGCAAAGGGAGAAAGTAGGAGATCTCTCTTGCGAGATGATCCCGCATTTTCTTG AAAGCTTTGCAGAGGCTAGCAGAATTACCCTCCACGTTGATTGTCTGCGAGGCAAGAATGATCATCACCG TAGTGAGAGTGCGTTCAAGGCTCTTGCGGTTGCCATAAGAGAAGCCACCTCGCCCAATGGTACCAACGAT GTTCCCTCCACCAAAGGTGTTCTTATGAGATCTCTCGAGTAATAGAGTCGAC-3 '

(BamHI site: GGATCC; original 5' leader: boxed region; MS2 stem-loop: in purple; HIS3( $\triangle \mathrm{AUG})$ : in green; XhoI site: CTCGAG; SalI site: GTCGAC)

B) BamHI site - original 5' leader - MS2 stem-loop - Luc2( $\triangle A \cup G)$ - Sall site 5' -GGATCCCTGCAGACATATACTGATACGATTGATCAGGTCTCGGTGAGGATCACCCATCGAAGATCC GAAGATGCCAAAAACATTAAGAAGGGCCCAGCGCCATTCTACCCACTCGAAGACGGGACCGCCGGCGAGC AGCTGCACAAAGCCATGAAGCGCTACGCCCTGGTGCCCGGCACCATCGCCTTTACCGACGCACATATCGA GGTGGACATTACCTACGCCGAGTACTTCGAGATGAGCGTTCGGCTGGCAGAAGCTATGAAGCGCTATGGG CTGAATACAAACCATCGGATCGTGGTGTGCAGCGAGAATAGCTTGCAGTTCTTCATGCCCGTGTTGGGTG CCCTGTTCATCGGTGTGGCTGTGGCCCCAGCTAACGACATCTACAACGAGCGCGAGCTGCTGAACAGCAT GGGCATCAGCCAGCCCACCGTCGTATTCGTGAGCAAGAAAGGGCTGCAAAAGATCCTCAACGTGCAAAAG AAGCTACCGATCATACAAAAGATCATCATCATGGATAGCAAGACCGACTACCAGGGCTTCCAAAGCATGT ACACCTTCGTGACTTCCCATTTGCCACCCGGCTTCAACGAGTACGACTTCGTGCCCGAGAGCTTCGACCG GGACAAAACCATCGCCCTGATCATGAACAGTAGTGGCAGTACCGGATTGCCCAAGGGCGTAGCCCTACCG CACCGCACCGCTTGTGTCCGATTCAGTCATGCCCGCGACCCCATCTTCGGCAACCAGATCATCCCCGACA CCGCTATCCTCAGCGTGGTGCCATTTCACCACGGCTTCGGCATGTTCACCACGCTGGGCTACTTGATCTG 
CGGCTTTCGgGTCGTGCTCATGTACCGCTTCGAGGAGGAGCTATTCTTGCGCAGCTTGCAAGACTATAAG АTTCAАTСTGСССTGCTGGTGCCCACACTATTTAGCTTCTTCGCTAAGAGCACTCTCATCGACAAGTACG АССТАAGCAАСTTGCACGAGATCGCCAGCGGCGGGGCGCCGCTCAGCAAGGAGGTAGGTGAGGCCGTGGC САAАCGCTTCCACCTACCAGGCATCCGCCAGGGCTACGGCCTGACAGAAACAACCAGCGCCATTCTGATC ACCCCCGAAGGGGACGACAAGCCTGGCGCAGTAGGCAAGGTGGTGCCCTTCTTCGAGGCTAAGGTGGTGG АСTTGGACACCGGTAAGACACTGGGTGTGAACCAGCGCGGCGAGCTGTGCGTCCGTGGCCCCATGATCAT GAGCGGCTACGTTAACAACCCCGAGGCTACAAACGCTCTCATCGACAAGGACGGCTGGCTGCACAGCGGC GACATCGCCTACTGGGACGAGGACGAGCACTTCTTCATCGTGGACCGGCTGAAGAGCCTGATCAAATACA AGGGCTACCAGGTAGCCCCAGCCGAACTGGAGAGCATCCTGCTGCAACACCCCAACATCTTCGACGCCGG GGTCGCCGGCCTGCCCGACGACGATGCCGGCGAGCTGCCCGCCGCAGTCGTCGTGCTGGAACACGGTAAA ACСАTGACCGAGAAGGAGATCGTGGACTATGTGGCCAGCCAGGTTACAACCGCCAAGAAGCTGCGCGGTG GTGTTGTGTTCTGGACGAGGTGCCTAAAGGACTGACCGGCAAGTTGGACGCCCGCAAGATCCGCGAGAT TСТСАTTAАGGCCAAGAAGGGCGGCAAGATCGCCGTGAGATCTCTCGAGTAATAGAGTCGAC-3 '

(BamHI site: GGATCC; original 5' leader: boxed region; MS2 stem-loop: in purple; Luc2( $\triangle \mathrm{AUG})$ : in yellow; XhoI site: CTCGAG; SalI site: GTCGAC)

\section{Text S2. The sequences of the ligand proteins used in this study.}

The genes encoding non-oligomeric MS2 coat protein ${ }^{35}$ and dCas 9 protein from $S$. pyrogenes used in this study are codon-optimized for S. cerevisiae and inserted into the EcoRI-SalI site of p414TEF and p413TEF, respectively. Yeast-codon optimized dCas9 is derived from pTGI_dCas $9^{34}$. Their sequences are as follows:

A) EcoRI site - MS2 coat protein gene - Sall site

5' -GAATTCGCTAGCCATATGCATACCATGGGATCCGCTTCTAATTTCACTCAATTCGTTTTGGTTGAC AACGGTGGTACTGGTGATGTTACTGTTGCTCCATCTAATTTTGCTAACGGTGTTGCTGAATGGATCTCC TCTAATTCAAGATCACAAGCCTACAAGGTTACCTGCTCTGTTAGACAATCTTCTGCTCAAAACAGAAAG TACACCATCAAGGTTGAAGTTCCAAAAGGTGCTTGGAGATCCTACTTGAATATGGAATTGACCATTCCA АTTTTCGCCACCAACTCTGATTGTGAATTGATCGTTAAGGCTATGCAAGGTTTGTTGAAGGATGGTAAT CСАATTCCATCTGCTATTGCTGCTAACTCCGGTATCTATAGATCTCTCGAGTAATAGAGTCGAC-3' (EcoRI: GAATTC; MS2 coat protein gene: boxed region; XhoI site: CTCGAG; SalI: GTCGAC) 
B) EcoRI site - dCas9 gene - Sall site

5 ' -GAATTCGCTAGCCAT ATGCATACCATGGGATCCGATAAGAAATACTCTATTGGTTTGGCTATCGGT ACAAACTCTGTTGGTTGGGCTGTTATTACTGATGAATACAAGGTTCCATCCAAGAAGTTCAAGGTTTTG GGTAACACTGATAGACACTCCATCAAAAAGAACTTGATTGGTGCCTTGTTGTTCGATTCTGGTGAAACT GCTGAAGCTACTAGATTGAAAAGAACCGCTAGAAGAAGATACACCAGAAGAAAGAACAGAATCTGCTAC TTGCAAGAAATCTTCTCCAACGAAATGGCCAAGGTTGATGATTCATTCTTCCACAGATTGGAAGAATCC TTCTTGGTCGAAGAAGATAAGAAGCACGAAAGACATCCAATCTTCGGTAACATCGTTGATGAAGTTGCT TACCACGAAAAGTACCCAACTATCTACCATTTGAGAAAGAAGTTGGTTGACTCTACCGATAAGGCTGAT TTGAGATTGATCTATTTGGCTTTGGCCCACATGATTAAGTTCAGAGGTCATTTCTTGATCGAAGGTGAT TTGAACCCAGATAACTCCGATGTTGATAAGTTGTTCATCCAATTAGTCCAAACCTACAATCAATTATTC GAAGAAAACCCAATCAACGCCTCTGGTGTTGATGCTAAAGCTATTTTGTCTGCCAGATTGTCCAAGTCC AGAAGATTAGAAAATTTGATCGCCCAATTACCAGGTGAAAAGAAGAATGGTTTGTTCGGTAATTTGATT GCCTTGTCTTTGGGTTTGACTCCAAACTTCAAGTCCAATTTCGATTTGGCTGAAGATGCCAAGTTGCAA TTATCTAAGGATACCTACGATGACGATTTGGATAACTTGTTGGCTCAAATCGGTGATCAATACGCTGAT TTGTTTTTGGCTGCTAAGAACTTGTCCGATGCCATTTTGTTGTCCGATATTTTGAGAGTCAACACCGAA ATTACTAAGGCTCCATTGTCTGCCTCTATGATCAAAAGATACGATGAACACCACCAAGACTTGACTTTG TTGAAGGCTTTGGTCAGACAACAATTACCTGAAAAGTACAAAGAAATTTTCTTCGATCAATCCAAGAAC GGTTACGCCGGTTATATTGATGGTGGTGCTTCTCAAGAAGAATTTTACAAGTTCATCAAGCCAATCTTG GAAAAGATGGACGGTACTGAAGAATTATTGGTCAAGTTGAACAGAGAAGATTTGTTGAGAAAGCAAAGA ACCTTCGACAACGGTTCTATTCCACATCAAATTCACTTGGGTGAATTGCACGCAATTTTGAGAAGACAA GAAGATTTTTATCCATTCTTGAAGGACAACAGAGAAAAGATCGAAAAGATTCTGACCTTCAGAATCCCT TACTACGTTGGTCCATTGGCTAGAGGTAATTCAAGATTTGCCTGGATGACTAGAAAGTCCGAAGAAACT ATTACTCCTTGGAACTTCGAAGAAGTTGTAGATAAGGGTGCTTCTGCCCAATCCTTTATTGAAAGAATG ACCAACTTCGACAAGAACTTGCCAAACGAAAAGGTTTTGCCAAAGCACTCTTTGTTGTACGAATACTTC ACCGTCTACAACGAATTGACTAAGGTTAAGTACGTCACCGAAGGTATGAGAAAACCAGCTTTTTTATCC GGTGAACAAAAGAAGGCTATCGTCGATTTGTTGTTCAAGACCAACAGAAAGGTTACTGTCAAGCAATTA AAAGAAGATTACTTCAAGAAAATCGAATGCTTCGACTCCGTTGAAATTTCTGGTGTCGAAGATAGATTC AATGCCTCTTTAGGTACTTACCATGACTTGTTGAAAATCATCAAGGACAAGGATTTCTTGGACAACGAA GAAAACGAAGATATTTTGGAAGATATTGTCTTGACATTGACCTTGTTTGAAGATAGAGAAATGATTGAA GAAAGATTGAAAACCTACGCCCACTTGTTCGATGATAAGGTTATGAAGCAATTAAAGAGAAGAAGATAC ACTGGTTGGGGTAGATTGTCCAGAAAATTGATTAACGGTATCAGAGACAAGCAATCCGGTAAGACCATT TTGGACTTTTTGAAGTCTGATGGTTTCGCTAACAGAAACTTCATGCAATTAATCCACGACGATTCCTTG 
ACTTTCAAAGAAGATATACAAAAGGCCCAAGTCTCTGGTCAAGGTGATTCTTTACATGAACATATCGCT AACTTGGCTGGTTCTCCAGCTATTAAGAAGGGTATTTTACAAACCGTTAAGGTCGTTGACGAATTGGTC AAAGTTATGGGTAGACATAAGCCAGAAAACATCGTTATCGAAATGGCTAGAGAAAATCAAACCACCCAA AAGGGTCAAAAGAACTCCAGAGAAAGAATGAAGAGAATCGAAGAAGGTATCAAAGAATTGGGTTCCCAA ATTTTGAAAGAACACCCAGTTGAAAACACCCAATTACAAAACGAAAAGTTGTACTTGTACTACTTGCAA AACGGTAGAGATATGTACGTTGACCAAGAATTGGACATCAACAGATTGTCTGATTACGATGTTGACGCT ATCGTTCCACAATCTTTTTTGAAGGATGACTCCATTGACAACAAGGTCTTGACTAGATCCGATAAGAAT AGAGGTAAGTCCGATAACGTTCCATCTGAAGAAGTCGTTAAGAAAATGAAGAACTATTGGAGACAATTA TTGAACGCCAAGTTGATCACCCAAAGAAAGTTTGACAATTTGACCAAGGCTGAAAGAGGTGGTTTGTCT GAATTGGATAAGGCAGGTTTCATCAAAAGACAATTAGTAGAAACCAGACAAATCACCAAGCACGTTGCT CAAATTTTGGATAGTAGAATGAACACTAAGTACGACGAAAACGACAAATTGATCAGAGAAGTTAAGGTC ATTACCTTGAAGTCCAAGTTGGTTTCCGATTTCAGAAAGGACTTCCAATTCTACAAGGTCAGAGAAATC AACAACTACCATCATGCACATGATGCTTACTTGAATGCTGTTGTTGGTACTGCCTTGATTAAGAAGTAT CCAAAGTTGGAATCCGAATTTGTCTACGGTGATTACAAGGTTTACGACGTTAGAAAGATGATCGCCAAG TCCGAACAAGAAATTGGTAAAGCTACTGCCAAATACTTCTTCTACTCCAATATTATGAATTTCTTTAAG ACCGAAATCACTTTGGCCAACGGTGAAATTAGAAAAAGACCATTGATTGAAACTAATGGTGAAACAGGT GAAATCGTTTGGGATAAGGGTAGAGATTTTGCCACTGTTAGAAAGGTATTGTCCATGCCACAAGTAAAC ATCGTCAAAAAGACCGAAGTTCAAACTGGTGGTTTCTCCAAAGAATCCATTTTGCCTAAGAGAAACTCC GATAAGTTGATCGCTAGAAAAAAAGACTGGGACCCAAAAAAGTACGGTGGTTTTGATTCTCCAACTGTT GCTTACTCTGTTTTGGTTGTTGCTAAGGTCGAAAAGGGTAAGAGTAAGAAGTTGAAGTCCGTCAAAGAA TTATTAGGTATCACTATCATGGAAAGATCCTCATTCGAAAAGAATCCTATCGACTTTTTGGAAGCCAAG GGTTACAAAGAAGTCAAGAAGGACTTGATCATTAAGTTGCCAAAGTACAGTTTGTTCGAATTGGAAAAT GGTAGAAAGAGAATGTTGGCTTCTGCCGGTGAATTACAAAAGGGTAATGAATTGGCTTTGCCATCCAAG TACGTTAATTTCTTATACTTGGCCTCCCACTACGAAAAATTGAAAGGTTCTCCTGAAGATAACGAACAA AAGCAATTATTTGTCGAACAACACAAGCACTACTTGGACGAAATCATTGAACAAATTTCCGAATTTTCC AAAAGAGTCATTTTGGCTGACGCCAATTTGGACAAAGTTTTGTCAGCTTACAACAAGCACAGAGATAAG CCAATTAGAGAACAAGCTGAAAACATCATTCACTTGTTCACTTTGACTAACTTGGGTGCTCCAGCTGCT TTTAAGTATTTCGATACCACTATCGACAGAAAGAGATACACCTCTACCAAAGAAGTTTTGGACGCTACT TTGATCCACCAATCTATTACTGGTTTGTACGAAACTAGAATCGACTTGTCTCAATTAGGTGGTGATAGA TCTCTCGAGTAATAGAGTCGAC-3'

(EcoRI: GAATTC; dCas9 gene: boxed region; XhoI site: CTCGAG; SalI: GTCGAC) 
Text S3. The sequence of C-terminal protein tag fused in Figure 3E and 3F.

The DNA fragment encoding $6 \times$ His-tag and $3 \times$ FLAG-tag were amplified via PCR, digested with SalI and $X h o I$, and cloned into the $X h o I-X h o I$ site of the expression vectors used in Figure 2 (Text S2). The sequence of the fragment is as follows:

5'-gaGTCGACcatcatcaccatcaccac GACTATAAGGACCATGACGGTGATTATAAAGATCATGACA TCGACTACAAGGATGACGATGACAAGTAACTCGAGTCATGTAATTAGTTATGTCACGCTTACATTCACG CССTCCCCCCACATCCGCTCTAACCGAAAAGGAAGGAGTTAGACAACCTGAAGTCTAGGTCCCTATTTAT TTTTTTATAGTTATGTTAGTATTAAGAACGTTATTTATATTTCAAATTTTTCTTTTTTTTCTGTACAGAC GCGTGTACGCATGTAACATTATACTGAAAACCTTGCTTGAGAAGGTTTTGGGACGCTCGAAGGCTTTAAT TTGCGGCCGGTACCCaattcgccctatagtgagtcgtattacgcgcgctcactggccgtcgttttacaac gtcgtgactgggaaaaccct-3'

(SalI site: GTCGAC; $6 \times$ His-tag: shaded region; 3×FLAG: boxed region; XhoI site: CTCGAG)

Text S4. The 5' leader sequence of the dCas9-driven non-AUG ON switch w/o NCC.

The DNA fragment of the synthetic leader sequence with the variant sgRNA was fused with the 5' end of Luc2 ( $\triangle \mathrm{AUG})$. The sequence of the fragment is as follows:

5'-GGATCCaCCTCTAACаCCTCTAACаCCTCCAACасCTCTAACaCCTCTAACaCCTCTaacacctcta acacctctaacAC GTTTTtGAGCaAGAAATtGCAAGTaAAAATAAGGCTAGTCCGTTATCAACTTGAAA AAGTGGCACCGAGTCGGTGC GAAGATGCCAAAAACATTAAGAAGG......GGCAAGATCGCCGTGAGATCTC TCGAGTAATAGAGTCGAC-3'

(BamHI: GGATCC; leader sequence: underlined; antitarget sequence: shaded; sgRNA variant: boxed; the part of Luc2: in yellow; SalI site: GTCGAC)

\section{Reference for Supporting Text}

(35) Peabody, D. S., and Ely, K. R. (1992) Control of translational repression by protein-protein interactions. Nucleic Acids Res. 20, 1649-1655. 
A
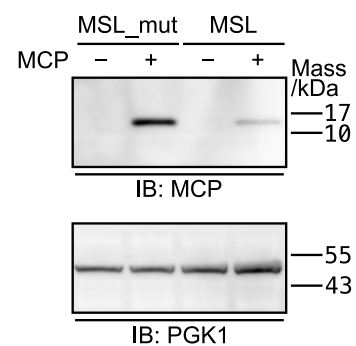

B

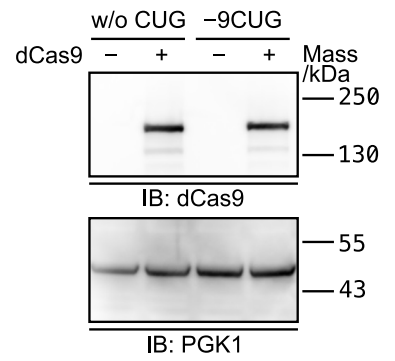

Figure S1. Western blotting analysis of trans-acting ligand proteins.

(A) Detection of the MS2 coat protein from the yeast transformants used in Figure 2B. MCP, $13.6 \mathrm{kDa}$; PGK1, $44.7 \mathrm{kDa}$. (B) Detection of dCas9 from the yeast transformants used in Figure 5C and D. dCas9, $159.3 \mathrm{kDa}$; PGK1, $44.7 \mathrm{kDa}$.
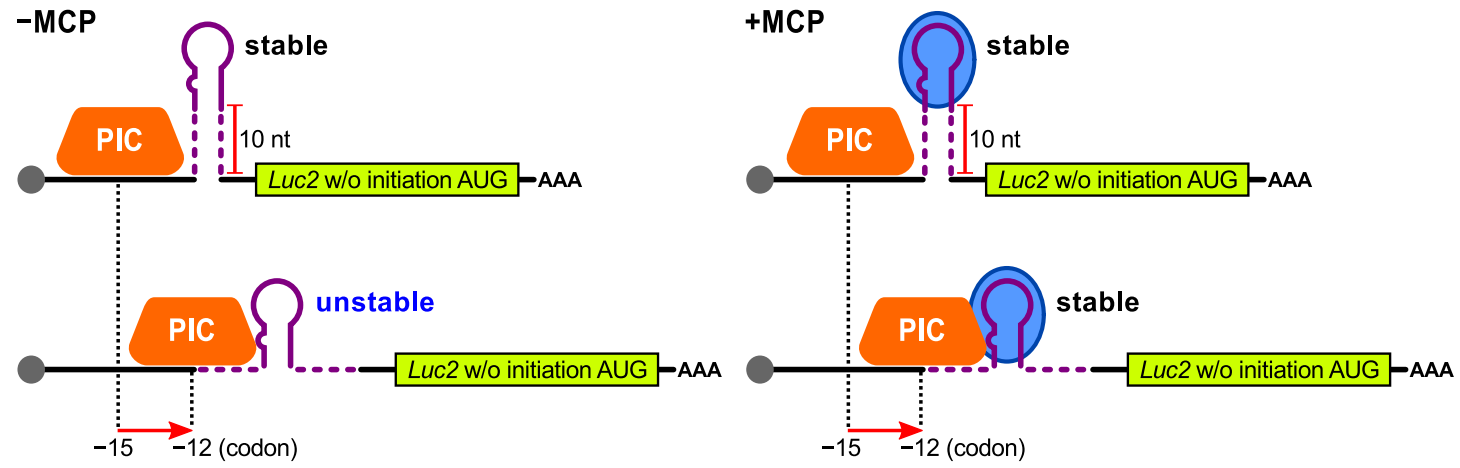

Figure S2. The working model for the MCP-driven non-AUG ON switch.

Schematic illustration of two equivalent forms of the switch in the absence (left) and presence of MCP. The upper form is more stable and triggers non-AUG initiation even in the absence of MCP. On the contrary, the lower form does not trigger non-AUG initiation in the absence of MCP but does in its presence. The numbers at the bottom indicate the position of the putative initiation codon in the 5 ' leader sequence, as shown in Figure 4A. MCP, MS2 coat protein; PIC, preinitiation complex. 


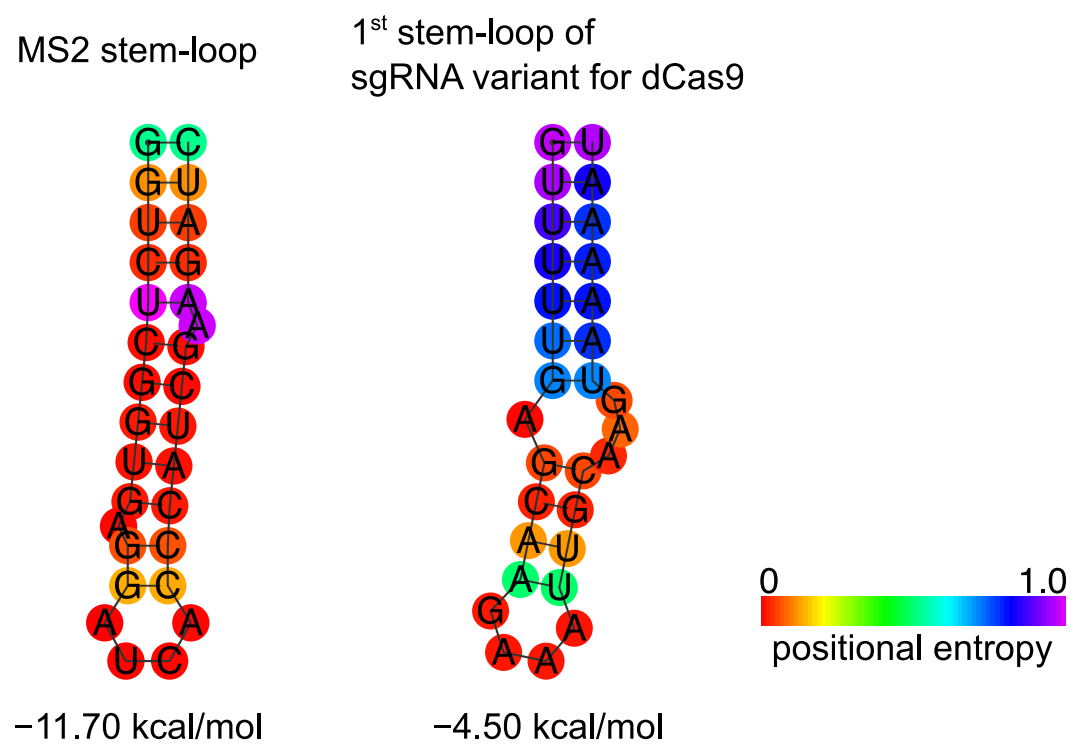

Figure S3. Predicted secondary structure and thermostability of the MS2 stem-loop and the sgRNA for dCas9 used in this study.

The RNA secondary structures of "AUG”-less MS2 stem-loop and the variant sgRNA used as an aptamer of the non-AUG ON switches were predicted by RNAfold WebServer (http://rna.tbi.univie.ac.at/cgibin/RNAWebSuite/RNAfold.cgi). The calculated minimum free energy and positional entropy are shown. 
Table S1. List of plasmid sets used in this study.

\begin{tabular}{|c|c|c|c|c|c|}
\hline Fig. & \# & Label_1 & Label_2 & Plasmid_1 & Plasmid_2 \\
\hline $2 \mathrm{~B}$ & 1 & $-\mathrm{MCP}$ & MSL & p414TEF & p6T-original-MSL-HIS3 \\
\hline $2 \mathrm{~B}$ & 2 & $+\mathrm{MCP}$ & MSL & $\mathrm{p} 4 \mathrm{~T}-\mathrm{MS} 2 \mathrm{CP}$ & p6T-original-MSL-HIS3 \\
\hline $2 \mathrm{~B}$ & 3 & $-\mathrm{MCP}$ & MSL_mut & p414TEF & p6T-original-MSL_mut-HIS3 \\
\hline $2 \mathrm{~B}$ & 4 & $+\mathrm{MCP}$ & MSL_mut & $\mathrm{p} 4 \mathrm{~T}-\mathrm{MS} 2 \mathrm{CP}$ & p6T-original-MSL_mut-HIS3 \\
\hline $2 \mathrm{C}$ & 1 & $-\mathrm{MCP}$ & MSL & p414TEF & p6T-original-MSL-Luc2 \\
\hline $2 \mathrm{C}$ & 2 & $+\mathrm{MCP}$ & MSL & $\mathrm{p} 4 \mathrm{~T}-\mathrm{MS} 2 \mathrm{CP}$ & p6T-original-MSL-Luc2 \\
\hline $2 \mathrm{C}$ & 3 & $-\mathrm{MCP}$ & MSL_mut & p414TEF & p6T-original-MSL_mut-Luc2 \\
\hline $2 \mathrm{C}$ & 4 & $+\mathrm{MCP}$ & MSL_mut & $\mathrm{p} 4 \mathrm{~T}-\mathrm{MS} 2 \mathrm{CP}$ & p6T-original-MSL_mut-Luc2 \\
\hline $3 \mathrm{~B}$ & 1 & $-\mathrm{MCP}$ & original & $\mathrm{p} 414 \mathrm{TEF}$ & p6T-original-MSL-HIS3 \\
\hline $3 B$ & 2 & $-\mathrm{MCP}$ & $-9 \mathrm{CUU}$ & p414TEF & p6T--9CUU-MSL-HIS3 \\
\hline $3 \mathrm{~B}$ & 3 & $-\mathrm{MCP}$ & $-3 A C C$ & $\mathrm{p} 414 \mathrm{TEF}$ & p6T--3ACC-MSL-HIS3 \\
\hline $3 B$ & 4 & $-\mathrm{MCP}$ & $-2 \mathrm{CUU}$ & p414TEF & p6T--2CUU-MSL-HIS3 \\
\hline $3 B$ & 5 & $-\mathrm{MCP}$ & $-9 C U U \&-3 A C C$ & $\mathrm{p} 414 \mathrm{TEF}$ & p6T--9CUU_-3ACC-MSL-HIS3 \\
\hline $3 B$ & 6 & $-\mathrm{MCP}$ & $-3 A C C \&-2 C U U$ & $\mathrm{p} 414 \mathrm{TEF}$ & p6T--3ACC_-2CUU-MSL-HIS3 \\
\hline $3 B$ & 7 & $-\mathrm{MCP}$ & $-3 A C C+C$ & p414TEF & $\mathrm{p} 6 \mathrm{~T}--3 \mathrm{ACC}+\mathrm{C}-\mathrm{MSL}-\mathrm{HIS} 3$ \\
\hline $3 \mathrm{~B}$ & 8 & $+\mathrm{MCP}$ & original & $\mathrm{p} 4 \mathrm{~T}-\mathrm{MS} 2 \mathrm{CP}$ & p6T-original-MSL-HIS3 \\
\hline $3 B$ & 9 & $+\mathrm{MCP}$ & $-9 \mathrm{CUU}$ & $\mathrm{p} 4 \mathrm{~T}-\mathrm{MS} 2 \mathrm{CP}$ & p6T--9CUU-MSL-HIS3 \\
\hline $3 B$ & 10 & $+\mathrm{MCP}$ & $-3 \mathrm{ACC}$ & $\mathrm{p} 4 \mathrm{~T}-\mathrm{MS} 2 \mathrm{CP}$ & p6T--3ACC-MSL-HIS3 \\
\hline $3 B$ & 11 & $+\mathrm{MCP}$ & $-2 \mathrm{CUU}$ & $\mathrm{p} 4 \mathrm{~T}-\mathrm{MS} 2 \mathrm{CP}$ & p6T--2CUU-MSL-HIS3 \\
\hline $3 B$ & 12 & $+\mathrm{MCP}$ & $-9 \mathrm{CUU} \&-3 \mathrm{ACC}$ & $\mathrm{p} 4 \mathrm{~T}-\mathrm{MS} 2 \mathrm{CP}$ & p6T--9CUU_-3ACC-MSL-HIS3 \\
\hline $3 B$ & 13 & $+\mathrm{MCP}$ & $-3 A C C \&-2 C U U$ & $\mathrm{p} 4 \mathrm{~T}-\mathrm{MS} 2 \mathrm{CP}$ & p6T--3ACC_-2CUU-MSL-HIS3 \\
\hline $3 B$ & 14 & $+\mathrm{MCP}$ & $-3 A C C+C$ & $\mathrm{p} 4 \mathrm{~T}-\mathrm{MS} 2 \mathrm{CP}$ & p6T--3ACC+C-MSL-HIS3 \\
\hline $3 C$ & 1 & $-\mathrm{MCP}$ & original & p414TEF & p6T-original-MSL-HIS3 \\
\hline $3 C$ & 2 & $-\mathrm{MCP}$ & $-10 \mathrm{UAA}$ & p414TEF & p6T--10UAA-MSL-HIS3 \\
\hline $3 C$ & 3 & $-\mathrm{MCP}$ & $-8 \mathrm{UAA}$ & $\mathrm{p} 414 \mathrm{TEF}$ & p6T--8UAA-MSL-HIS3 \\
\hline $3 C$ & 4 & $-\mathrm{MCP}$ & $-6 U A A$ & p414TEF & p6T--6UAA-MSL-HIS3 \\
\hline $3 C$ & 5 & $+\mathrm{MCP}$ & original & $\mathrm{p} 4 \mathrm{~T}-\mathrm{MS} 2 \mathrm{CP}$ & p6T-original-MSL-HIS3 \\
\hline $3 C$ & 6 & $+\mathrm{MCP}$ & $-10 \mathrm{UAA}$ & $\mathrm{p} 4 \mathrm{~T}-\mathrm{MS} 2 \mathrm{CP}$ & p6T--10UAA-MSL-HIS3 \\
\hline $3 C$ & 7 & $+\mathrm{MCP}$ & $-8 \mathrm{UAA}$ & $\mathrm{p} 4 \mathrm{~T}-\mathrm{MS} 2 \mathrm{CP}$ & p6T--8UAA-MSL-HIS3 \\
\hline $3 C$ & 8 & $+\mathrm{MCP}$ & $-6 \mathrm{UAA}$ & $\mathrm{p} 4 \mathrm{~T}-\mathrm{MS} 2 \mathrm{CP}$ & p6T--6UAA-MSL-HIS3 \\
\hline
\end{tabular}




\begin{tabular}{|c|c|c|c|c|c|}
\hline $3 \mathrm{E}$ & 1 & $-\mathrm{MCP}$ & CUG & $\mathrm{p} 414 \mathrm{TEF}$ & p6T-original-MSL-HIS3-FLAG \\
\hline $3 E$ & 2 & $+\mathrm{MCP}$ & CUG & $\mathrm{p} 4 \mathrm{~T}-\mathrm{MS} 2 \mathrm{CP}$ & p6T-original-MSL-HIS3-FLAG \\
\hline $3 \mathrm{E}$ & 3 & $-\mathrm{MCP}$ & CUU & $\mathrm{p} 414 \mathrm{TEF}$ & p6T--9CUU-MSL-HIS3-FLAG \\
\hline $3 E$ & 4 & $+\mathrm{MCP}$ & CUU & $\mathrm{p} 4 \mathrm{~T}-\mathrm{MS} 2 \mathrm{CP}$ & p6T--9CUU-MSL-HIS3-FLAG \\
\hline $3 E$ & 5 & $-\mathrm{MCP}$ & AUG & $\mathrm{p} 414 \mathrm{TEF}$ & p6T--9AUG-MSL-HIS3-FLAG \\
\hline $3 F$ & 1 & $-\mathrm{MCP}$ & CUG & $\mathrm{p} 414 \mathrm{TEF}$ & p6T-original-MSL-Luc2-FLAG \\
\hline $3 F$ & 2 & $+\mathrm{MCP}$ & CUG & $\mathrm{p} 4 \mathrm{~T}-\mathrm{MS} 2 \mathrm{CP}$ & p6T-original-MSL-Luc2-FLAG \\
\hline $3 F$ & 3 & $-\mathrm{MCP}$ & CUU & $\mathrm{p} 414 \mathrm{TEF}$ & p6T--9CUU-MSL-Luc2-FLAG \\
\hline $3 F$ & 4 & $+\mathrm{MCP}$ & CUU & $\mathrm{p} 4 \mathrm{~T}-\mathrm{MS} 2 \mathrm{CP}$ & p6T--9CUU-MSL-Luc2-FLAG \\
\hline $3 F$ & 5 & $-\mathrm{MCP}$ & AUG & $\mathrm{p} 414 \mathrm{TEF}$ & p6T--9AUG-MSL-Luc2-FLAG \\
\hline $4 \mathrm{~B}$ & 1 & $-\mathrm{MCP}$ & w/o CUG & $\mathrm{p} 414 \mathrm{TEF}$ & p6T-TSN-null-MSL-Luc2-FLAG \\
\hline $4 \mathrm{~B}$ & 2 & $-\mathrm{MCP}$ & $-21 \mathrm{CUG}$ & $\mathrm{p} 414 \mathrm{TEF}$ & p6T-TSN--21CUG-MSL-Luc2-FLAG \\
\hline $4 \mathrm{~B}$ & 3 & $-\mathrm{MCP}$ & $-18 \mathrm{CUG}$ & $\mathrm{p} 414 \mathrm{TEF}$ & p6T-TSN--18CUG-MSL-Luc2-FLAG \\
\hline $4 \mathrm{~B}$ & 4 & $-\mathrm{MCP}$ & $-15 \mathrm{CUG}$ & $\mathrm{p} 414 \mathrm{TEF}$ & p6T-TSN--15CUG-MSL-Luc2-FLAG \\
\hline $4 \mathrm{~B}$ & 5 & $-\mathrm{MCP}$ & -12 CUG & $\mathrm{p} 414 \mathrm{TEF}$ & p6T-TSN--12CUG-MSL-Luc2-FLAG \\
\hline $4 \mathrm{~B}$ & 6 & $-\mathrm{MCP}$ & -9 CUG & $\mathrm{p} 414 \mathrm{TEF}$ & p6T-TSN--9CUG-MSL-Luc2-FLAG \\
\hline $4 \mathrm{~B}$ & 7 & $-\mathrm{MCP}$ & $-6 \mathrm{CUG}$ & $\mathrm{p} 414 \mathrm{TEF}$ & p6T-TSN--6CUG-MSL-Luc2-FLAG \\
\hline $4 \mathrm{~B}$ & 8 & $-\mathrm{MCP}$ & -3 CUG & $\mathrm{p} 414 \mathrm{TEF}$ & p6T-TSN--3CUG-MSL-Luc2-FLAG \\
\hline $4 \mathrm{~B}$ & 9 & $+\mathrm{MCP}$ & w/o CUG & $\mathrm{p} 4 \mathrm{~T}-\mathrm{MS} 2 \mathrm{CP}$ & p6T-TSN-null-MSL-Luc2-FLAG \\
\hline $4 \mathrm{~B}$ & 10 & $+\mathrm{MCP}$ & $-21 \mathrm{CUG}$ & $\mathrm{p} 4 \mathrm{~T}-\mathrm{MS} 2 \mathrm{CP}$ & p6T-TSN--21CUG-MSL-Luc2-FLAG \\
\hline $4 \mathrm{~B}$ & 11 & $+\mathrm{MCP}$ & $-18 \mathrm{CUG}$ & $\mathrm{p} 4 \mathrm{~T}-\mathrm{MS} 2 \mathrm{CP}$ & p6T-TSN--18CUG-MSL-Luc2-FLAG \\
\hline $4 \mathrm{~B}$ & 12 & $+\mathrm{MCP}$ & $-15 \mathrm{CUG}$ & $\mathrm{p} 4 \mathrm{~T}-\mathrm{MS} 2 \mathrm{CP}$ & p6T-TSN--15CUG-MSL-Luc2-FLAG \\
\hline $4 \mathrm{~B}$ & 13 & $+\mathrm{MCP}$ & $-12 \mathrm{CUG}$ & $\mathrm{p} 4 \mathrm{~T}-\mathrm{MS} 2 \mathrm{CP}$ & p6T-TSN--12CUG-MSL-Luc2-FLAG \\
\hline $4 \mathrm{~B}$ & 14 & $+\mathrm{MCP}$ & -9 CUG & $\mathrm{p} 4 \mathrm{~T}-\mathrm{MS} 2 \mathrm{CP}$ & p6T-TSN--9CUG-MSL-Luc2-FLAG \\
\hline $4 \mathrm{~B}$ & 15 & $+\mathrm{MCP}$ & -6 CUG & $\mathrm{p} 4 \mathrm{~T}-\mathrm{MS} 2 \mathrm{CP}$ & p6T-TSN--6CUG-MSL-Luc2-FLAG \\
\hline $4 \mathrm{~B}$ & 16 & $+\mathrm{MCP}$ & -3 CUG & $\mathrm{p} 4 \mathrm{~T}-\mathrm{MS} 2 \mathrm{CP}$ & p6T-TSN--3CUG-MSL-Luc2-FLAG \\
\hline $5 C$ & 1 & $-\operatorname{dCas} 9$ & w/o CUG & $\mathrm{p} 413 \mathrm{TEF}$ & p6T-TSN-null-gRNA9-Luc2-FLAG \\
\hline $5 \mathrm{C}$ & 2 & $-\operatorname{dCas} 9$ & -15 CUG & $\mathrm{p} 413 \mathrm{TEF}$ & p6T-TSN--15CUG-gRNA9-Luc2-FLAG \\
\hline $5 \mathrm{C}$ & 3 & $-\operatorname{dCas} 9$ & -12 CUG & $\mathrm{p} 413 \mathrm{TEF}$ & p6T-TSN--12CUG-gRNA9-Luc2-FLAG \\
\hline $5 \mathrm{C}$ & 4 & $-\operatorname{dCas} 9$ & -9 CUG & $\mathrm{p} 413 \mathrm{TEF}$ & p6T-TSN--9CUG-gRNA9-Luc2-FLAG \\
\hline $5 \mathrm{C}$ & 5 & $-\operatorname{dCas} 9$ & -6 CUG & $\mathrm{p} 413 \mathrm{TEF}$ & p6T-TSN--6CUG-gRNA9-Luc2-FLAG \\
\hline $5 \mathrm{C}$ & 6 & $-\operatorname{dCas} 9$ & -3 CUG & $\mathrm{p} 413 \mathrm{TEF}$ & p6T-TSN--3CUG-gRNA9-Luc2-FLAG \\
\hline
\end{tabular}




\begin{tabular}{|c|c|c|c|c|c|}
\hline $5 \mathrm{C}$ & 7 & $-\mathrm{dCas} 9$ & OCUG & p413TEF & p6T-TSN-0CUG-gRNA9-Luc2-FLAG \\
\hline $5 \mathrm{C}$ & 8 & $-\mathrm{dCas} 9$ & +3 CUG & p413TEF & p6T-TSN-3CUG-gRNA9-Luc2-FLAG \\
\hline $5 \mathrm{C}$ & 9 & $+\mathrm{dCas} 9$ & w/o CUG & p3T-dCas 9 & p6T-TSN-null-gRNA9-Luc2-FLAG \\
\hline $5 \mathrm{C}$ & 10 & $+d \operatorname{das} 9$ & -15 CUG & p3T-dCas 9 & p6T-TSN--15CUG-gRNA9-Luc2-FLAG \\
\hline $5 \mathrm{C}$ & 11 & $+d \operatorname{das} 9$ & -12 CUG & p3T-dCas 9 & p6T-TSN--12CUG-gRNA9-Luc2-FLAG \\
\hline $5 \mathrm{C}$ & 12 & $+\mathrm{dCas} 9$ & -9 CUG & p3T-dCas 9 & p6T-TSN--9CUG-gRNA9-Luc2-FLAG \\
\hline $5 \mathrm{C}$ & 13 & $+\mathrm{dCas} 9$ & -6 CUG & p3T-dCas 9 & p6T-TSN--6CUG-gRNA9-Luc2-FLAG \\
\hline $5 C$ & 14 & $+\mathrm{dCas} 9$ & -3 CUG & p3T-dCas 9 & p6T-TSN--3CUG-gRNA9-Luc2-FLAG \\
\hline $5 \mathrm{C}$ & 15 & $+d \operatorname{dCas} 9$ & OCUG & p3T-dCas9 & p6T-TSN-0CUG-gRNA9-Luc2-FLAG \\
\hline $5 C$ & 16 & $+\mathrm{dCas} 9$ & +3 CUG & p3T-dCas 9 & p6T-TSN-3CUG-gRNA9-Luc2-FLAG \\
\hline $6 \mathrm{~B}$ & 1 & $-\mathrm{dCas} 9$ & $\mathrm{w} / \mathrm{O} \mathrm{NCC}$ & $\mathrm{p} 413 \mathrm{TEF}$ & p6T-TSN-null-gRNA9-Luc2-FLAG \\
\hline $6 \mathrm{~B}$ & 2 & $-\mathrm{dCas} 9$ & CUG & p413TEF & p6T-TSN--9CUG-gRNA9-Luc2-FLAG \\
\hline $6 \mathrm{~B}$ & 3 & $-\mathrm{dCas} 9$ & AUG & p413TEF & p6T-TSN--9AUG-gRNA9-Luc2-FLAG \\
\hline $6 \mathrm{~B}$ & 4 & $-\mathrm{dCas} 9$ & UUG & p413TEF & p6T-TSN--9UUG-gRNA9-Luc2-FLAG \\
\hline $6 \mathrm{~B}$ & 5 & $-\mathrm{dCas} 9$ & GUG & p413TEF & p6T-TSN--9GUG-gRNA9-Luc2-FLAG \\
\hline $6 \mathrm{~B}$ & 6 & $-\mathrm{dCas} 9$ & AUA & p413TEF & p6T-TSN--9AUA-gRNA9-Luc2-FLAG \\
\hline $6 \mathrm{~B}$ & 7 & $-\mathrm{dCas} 9$ & AUU & p413TEF & p6T-TSN--9AUU-gRNA9-Luc2-FLAG \\
\hline $6 \mathrm{~B}$ & 8 & $-\mathrm{dCas} 9$ & AUC & p413TEF & p6T-TSN--9AUC-gRNA9-Luc2-FLAG \\
\hline $6 \mathrm{~B}$ & 9 & $-\mathrm{dCas} 9$ & AAG & p413TEF & p6T-TSN--9AAG-gRNA9-Luc2-FLAG \\
\hline $6 \mathrm{~B}$ & 10 & $-\mathrm{dCas} 9$ & AGG & p413TEF & p6T-TSN--9AGG-gRNA9-Luc2-FLAG \\
\hline $6 \mathrm{~B}$ & 11 & $-\mathrm{dCas} 9$ & ACG & p413TEF & p6T-TSN--9ACG-gRNA9-Luc2-FLAG \\
\hline $6 \mathrm{~B}$ & 12 & $+\mathrm{dCas} 9$ & $\mathrm{w} / \mathrm{O} \mathrm{NCC}$ & p3T-dCas 9 & p6T-TSN-null-gRNA9-Luc2-FLAG \\
\hline $6 \mathrm{~B}$ & 13 & $+d \operatorname{dCas} 9$ & CUG & p3T-dCas 9 & p6T-TSN--9CUG-gRNA9-Luc2-FLAG \\
\hline $6 \mathrm{~B}$ & 14 & $+d \operatorname{dCas} 9$ & AUG & p3T-dCas 9 & p6T-TSN--9AUG-gRNA9-Luc2-FLAG \\
\hline $6 \mathrm{~B}$ & 15 & $+d \operatorname{dCas} 9$ & UUG & p3T-dCas 9 & p6T-TSN--9UUG-gRNA9-Luc2-FLAG \\
\hline $6 \mathrm{~B}$ & 16 & $+d \operatorname{das} 9$ & GUG & p3T-dCas 9 & p6T-TSN--9GUG-gRNA9-Luc2-FLAG \\
\hline $6 \mathrm{~B}$ & 17 & $+d \operatorname{Cas} 9$ & AUA & p3T-dCas 9 & p6T-TSN--9AUA-gRNA9-Luc2-FLAG \\
\hline $6 \mathrm{~B}$ & 18 & $+d \operatorname{dCas} 9$ & AUU & p3T-dCas 9 & p6T-TSN--9AUU-gRNA9-Luc2-FLAG \\
\hline $6 \mathrm{~B}$ & 19 & $+\mathrm{dCas} 9$ & AUC & p3T-dCas 9 & p6T-TSN--9AUC-gRNA9-Luc2-FLAG \\
\hline $6 \mathrm{~B}$ & 20 & $+d \operatorname{dCas} 9$ & AAG & p3T-dCas 9 & p6T-TSN--9AAG-gRNA9-Luc2-FLAG \\
\hline $6 \mathrm{~B}$ & 21 & $+d \operatorname{dCas} 9$ & AGG & p3T-dCas 9 & p6T-TSN--9AGG-gRNA9-Luc2-FLAG \\
\hline $6 \mathrm{~B}$ & 22 & $+\mathrm{dCas} 9$ & ACG & p3T-dCas 9 & p6T-TSN--9ACG-gRNA9-Luc2-FLAG \\
\hline
\end{tabular}


Table S2. The primers used for constructing the non-AUG ON switches in Figure 4.

\begin{tabular}{|c|c|}
\hline Primer name & Sequence ${ }^{1,2}$ \\
\hline TSN_nul1-MSL_F2 & 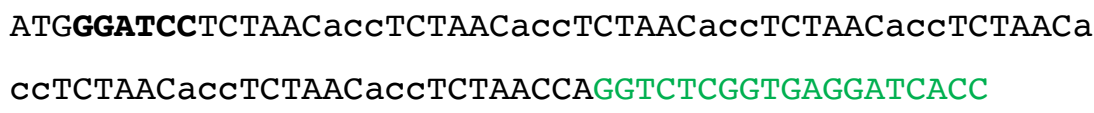 \\
\hline TSN_-3CUG-MSL_F2 & 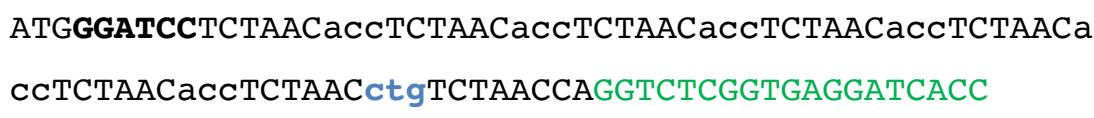 \\
\hline TSN_-6CUG-MSL_F2 & 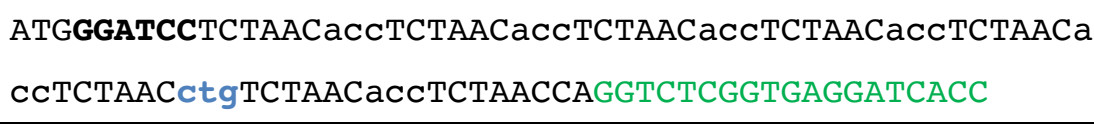 \\
\hline TSN_-9CUG-MSL_F2 & 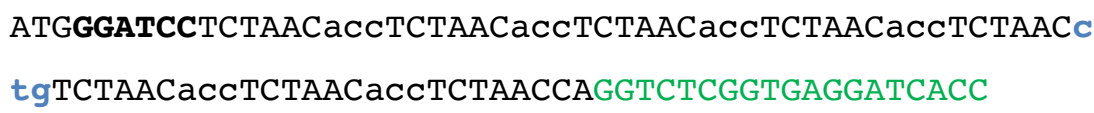 \\
\hline TSN_-12CUG-MSL_F2 & 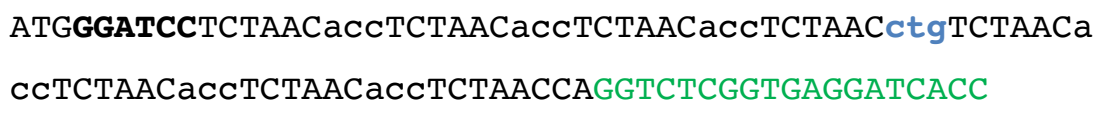 \\
\hline TSN_-15CUG-MSL_F2 & 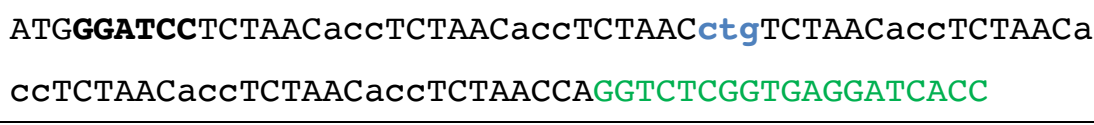 \\
\hline TSN_-18CUG-MSL_F2 & 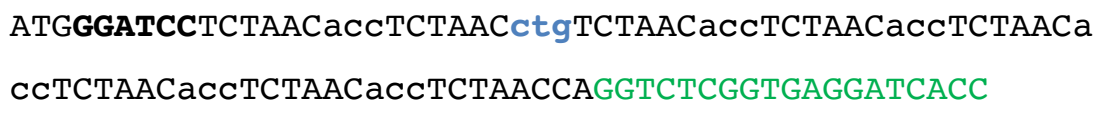 \\
\hline TSN_-21CUG-MSL_F 2 & 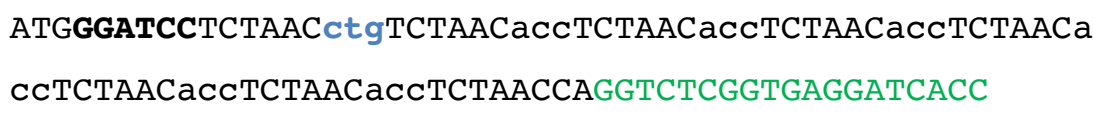 \\
\hline
\end{tabular}

${ }^{1}$ GGATCC, BamHI site.

${ }^{2}$ CUG non-AUG initiation codon and the 5' half of MSL are shown in blue small letters and in green capital letters, respectively. 
Table S3. The primers used for constructing the non-AUG ON switches in Figures 5 and 6.

\begin{tabular}{|c|c|}
\hline Primer name & Sequence ${ }^{1,2}$ \\
\hline g9_TSN_null_F2 & 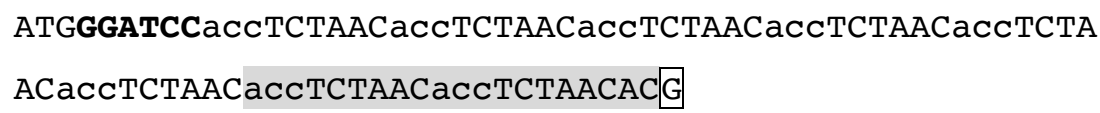 \\
\hline g9_TSN_3CUG_F 2 & 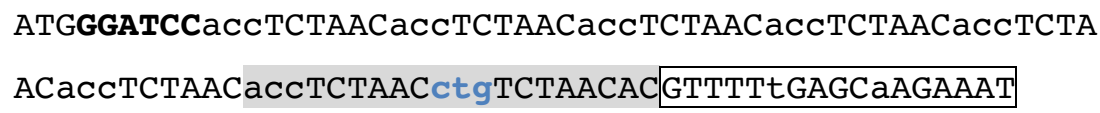 \\
\hline g9_TSN_0CUG_F2 & 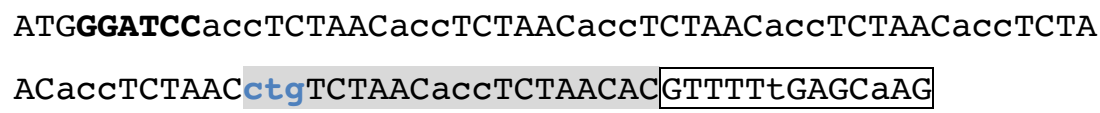 \\
\hline g9_TSN_-3CUG_F2 & 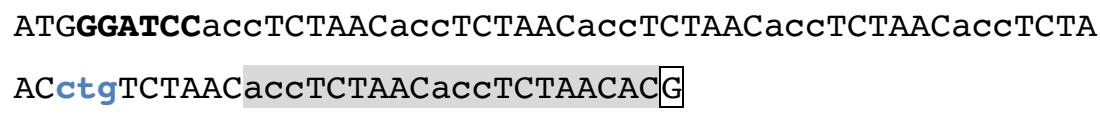 \\
\hline g9_TSN_-6CUG_F2 & 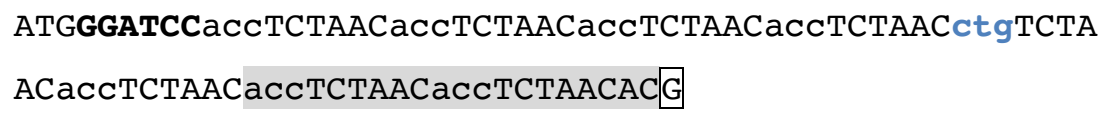 \\
\hline g9_TSN_-9CUG_F2 & 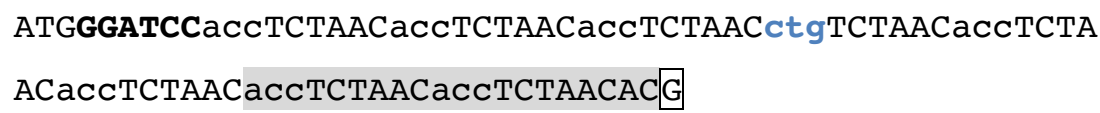 \\
\hline g9_TSN_-12CUG_F2 & 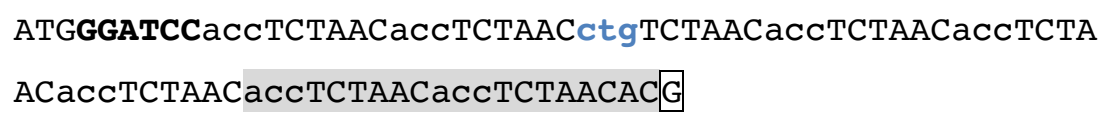 \\
\hline g9_TSN_-15CUG_F2 & 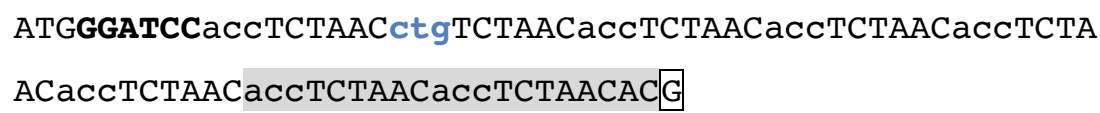 \\
\hline g9_TSN_-9AUG_F2 & 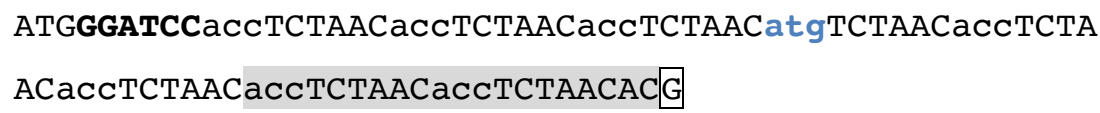 \\
\hline g9_TSN_-9UUG_F2 & 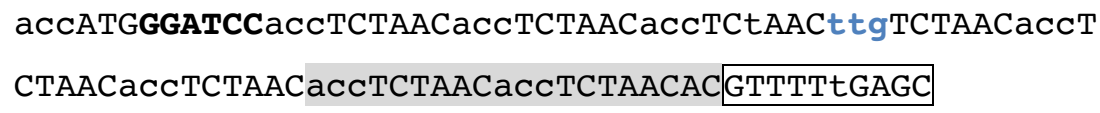 \\
\hline g9_TSN_-9GUG_F2 & 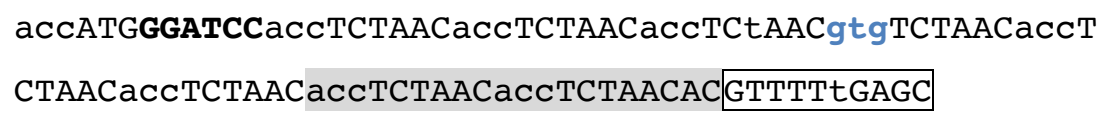 \\
\hline g9_TSN_-9AUA_F2 & 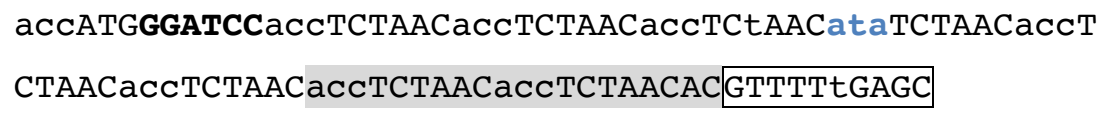 \\
\hline g9_TSN_-9AUU_F2 & 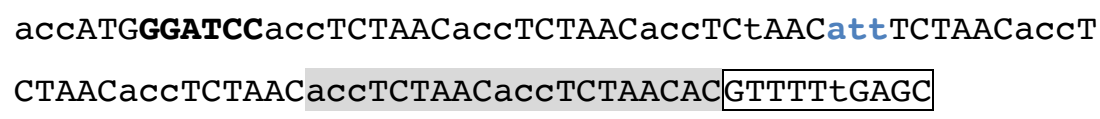 \\
\hline g9_TSN_-9AUC_F2 & 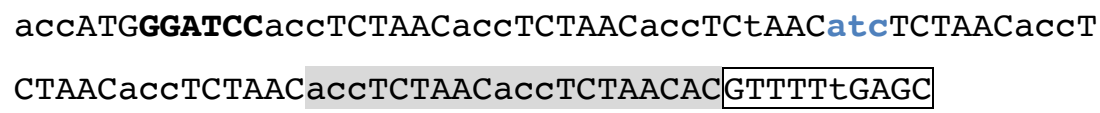 \\
\hline g9_TSN_-9AAG_F2 & 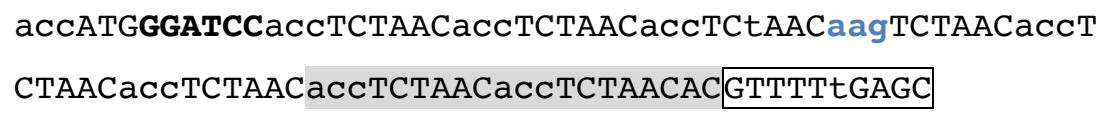 \\
\hline
\end{tabular}




\begin{tabular}{|l|l|}
\hline 99_TSN_-9AGG_F2 & $\begin{array}{l}\text { acCATGGGATCCaCCTCTAACaCCTCTAACaCCTCtAACaggTCTAACacCT } \\
\text { CTAACacCTCTAACacCTCTAACacCTCTAACACGTTTTtGAGC }\end{array}$ \\
\hline g9_TSN_-9ACG_F2 & $\begin{array}{l}\text { acCATGGGATCCaCCTCTAACaCCTCTAACaCCTCtAACacgTCTAACacCT } \\
\text { CTAACacCTCTAACacCTCTAACacCTCTAACACGTTTTtGAGC }\end{array}$ \\
\hline
\end{tabular}

${ }^{1}$ GGATCC, BamHI site; shaded, the antitarget sequence; boxed, the 5' part of the variant sgRNA.

${ }^{2}$ Non-AUG initiation codons are shown in blue small letters. 